Article

\title{
ILC with Initial State Learning for Fractional Order Linear Distributed Parameter Systems
}

\author{
Yong-Hong Lan * and Zhe-Min Cui \\ School of Information Engineering, Xiangtan University, Xiangtan 411105, China; zmincui@163.com \\ * Correspondence: yhlan@xtu.edu.cn; Tel.: +86-136-6742-0066
}

Received: 21 April 2018; Accepted: 9 June 2018; Published: 14 June 2018

\begin{abstract}
This paper presents a second order P-type iterative learning control (ILC) scheme with initial state learning for a class of fractional order linear distributed parameter systems. First, by analyzing the control and learning processes, a discrete system for P-type ILC is established, and the ILC design problem is then converted to a stability problem for such a discrete system. Next, a sufficient condition for the convergence of the control input and the tracking errors is obtained by introducing a new norm and using the generalized Gronwall inequality, which is less conservative than the existing one. Finally, the validity of the proposed method is verified by a numerical example.
\end{abstract}

Keywords: iterative learning control (ILC); fractional order; distributed parameter system; generalized gronwall inequality

\section{Introduction}

Iterative learning control (ILC) is an effective technique for improving the performance of systems that operate repetitively over a fixed time interval [1-4]. The fundamental idea in ILC is that the task to be carried out by the system is of a repetitive nature. By using such a repetitive nature, it is possible to adjust the input signal such that the output signal follows the reference signal as closely as possible. Owing to its simplicity and effectiveness, ILC is playing an important role in many areas and applications [5-7].

Distributed parameter systems are a class of complicated infinite-dimensional systems, whose states depend on both spatial position and time [8-11]. In recent years, the application of ILC to distributed parameter systems has become a new topic [12-15]. In [12], an open-loop P-type iterative learning controller was designed for the first order hyperbolic distributed parameter system. The P-type and D-type ILC algorithms were studied in [13] for a class of parabolic distributed parameter systems. For a class of single-input single-output coupling nonlinear distributed parameter systems, a P-type learning controller was designed, and the convergence conditions, speed and robustness of the iterative learning algorithm were also discussed in [14]. For uncertain nonlinear distributed parameter systems and state time-delay distributed parameter systems, the P-type ILC was investigated in $[15,16]$, respectively. A D-type anticipatory ILC scheme was applied to the boundary control of a class of inhomogeneous heat equations in [17]. In [18], a framework in the frequency domain of ILC was designed for linear inhomogeneous distributed parameter systems. By using the Gronwall-Bellman inequality, a close-loop P-type iterative learning algorithm is proposed for linear parabolic distributed parameter systems in [19]. In addition, based on the eigenspectrum and reduced order model, the ILC was proposed for a class of parabolic distributed parameter systems in [20].

Note that the above existing ILC methods for distributed parameter systems only focus on the lower order ILC. That is, to update the input profile, the learning algorithms use only the information from the last cycle. As we all known, the higher order learning algorithm incorporates the information not only from the last cycle, which can lead to better performance in terms of both robustness and 
convergence rate [21-23]. In [24], for a class of parabolic linear distributed parameter systems with uncertainty coefficients, an ILC algorithm with a forgetting factor was proposed, and the conditions for the convergence of the algorithm were established.

On the other hand, in recent years, fractional order control systems have attracted increasing interest. It was found that many systems in interdisciplinary fields could be elegantly described with the help of fractional derivatives and integrals, such as viscoelastic systems, dielectric polarization, electrode-electrolyte polarization and electromagnetic waves [25-27]. In [28], a fractional order distributed parameter system was used to describe radial ground water flow. The existence and uniqueness of mild and classical solutions for a class of distributed order fractional differential equations had been studied in [29]. By using the differential inequality method, some sufficient conditions for the oscillation of solutions for partial fractional differential equations were presented in [30]. A fundamental solution of a fractional order distributed parameter system was presented in [31]. The numerical solutions of such systems were proposed in the literature by means of finite difference methods [32], spectral collocation methods and others [33,34]. However, it is worth pointing out that up to now, there is no concern about ILC for fractional order distributed parameter systems.

The purpose of this paper is to present a second order P-type ILC algorithm with initial state learning for fractional order linear distributed parameter systems. The main contributions of this paper are as follows. (1) By analyzing the control and learning processes, the second order P-type ILC design problem for fractional order linear distributed parameter systems is converted to a stability problem for a discrete system. (2) Using the generalized Gronwall inequality, the control input and the tracking errors convergence analysis are given in detail. The obtained convergence condition is less conservative than some existing ones in recent literature in the case of integral order. (3) The proposed design method can be extended to ILC for fractional order parabolic distributed parameter systems involving time-delay, locally Lipschitz, as well as bounded external disturbance.

The rest of this paper is organized as follows: In Section 2, the problem formulation and some preliminaries are presented. The convergence analysis for the second order p-type ILC algorithm is derived in Section 3. Section 4 presents a numerical example that demonstrates the effectiveness of the method. Finally, some conclusions are drawn in Section 5.

\section{Preliminaries and Problem Statement}

In this section, some basic definitions and properties (for more details, see [25]) are first introduced, which will be used in the following sections.

Definition 1 ([25]). The definition of the Liouville fractional integral is described by:

$$
t_{0} I_{t}^{\alpha} f(t)=\frac{1}{\Gamma(\alpha)} \int_{t_{0}}^{t}(t-\tau)^{\alpha-1} f(\tau) d \tau, \quad \alpha>0,
$$

where $\Gamma(\cdot)$ is the well-known Gamma function, which is defined by $\Gamma(z)=\int_{0}^{\infty} e^{-z} t^{z-1} d t$.

Definition 2 ([25]). The Caputo derivative is defined by:

$$
{ }_{t_{0}}^{C} \mathcal{D}_{t}^{\alpha} f(t)=\frac{1}{\Gamma(n-\alpha)} \int_{t_{0}}^{t}(t-\tau)^{-\alpha+n-1} f^{(n)}(\tau) d \tau,
$$

where $n$ is the first integer, which is not less than $\alpha$, i.e., $\alpha \in[n-1, n)$.

In the following, for simplicity, we denote the fractional integral $t_{0} I_{t}^{\alpha}$ by $I^{\alpha}$ and the Caputo derivative ${ }_{t_{0}}^{C} \mathcal{D}_{t}^{\alpha}$ by $\mathcal{D}_{t}^{\alpha}$, respectively. 
Definition 3 ([25]). The two-parameter Mittag-Leffler function is defined by:

$$
E_{\alpha, \beta}(z)=\sum_{k=0}^{\infty} \frac{z^{k}}{\Gamma(\alpha k+\beta)}, \quad \alpha>0, \beta>0 .
$$

For $\beta=1, E_{\alpha, \beta}(z)=E_{\alpha}(z)$. Especially, $E_{1,1}(z)=e^{z}$.

To proceed with the discussion of the main results, the following lemmas are given.

Lemma 1 ([25]). From the definition of the Caputo derivative, when $0<\alpha \leq 1$, we have:

$$
I_{t}^{\alpha}\left(\mathcal{D}_{t}^{\alpha}(x(t))=x(t)-x\left(t_{0}\right) .\right.
$$

Lemma 2 ([35]). Let $x(t) \in \mathbb{R}^{n}$ be a vector of a differentiable function. Then, for any time instant $t \geq t_{0}$, the following relationship holds:

$$
\frac{1}{2} \mathcal{D}_{t}^{\alpha}\left(x^{T}(t) P x(t)\right) \leq x^{T}(t) P \mathcal{D}_{t}^{\alpha} x(t), \forall \alpha \in(0,1],
$$

where $P \in \mathbb{R}^{n \times n}$ is a constant, square, symmetric and positive definite matrix.

Lemma 3 ([25]). The fractional order differentiation or integral of the Mittag-Leffler function is:

$$
t_{0} \mathcal{D}_{t}^{\rho}\left[t^{\beta-1} E_{\alpha, \beta}\left(\lambda t^{\alpha}\right)\right]=t^{\beta-\rho-1} E_{\alpha, \beta-\rho}\left(\lambda t^{\alpha}\right),
$$

where $\rho<\beta$.

Lemma 4 (Generalized Gronwall inequality [36]). Suppose $\alpha>0, a(t)$ is a nonnegative, nondecreasing function locally integrable on $0 \leq t \leq T$ (some $T \leq+\infty$ ) and $g(t)$ is a nonnegative, nondecreasing continuous function defined on $0 \leq t \leq T, g(t) \leq M$ (constant), and suppose $u(t)$ is nonnegative and locally integrable on $0 \leq t \leq T$ with:

$$
u(t) \leq a(t)+g(t) \int_{0}^{t}(t-s)^{\alpha-1} u(s) d s,
$$

on the interval. Then:

$$
u(t) \leq a(t) E_{\alpha}\left(g(t) \Gamma(\alpha) t^{\alpha}\right) .
$$

In this paper, we consider a class of fractional order linear distributed parameter systems with repetitive operation characteristics as follows:

$$
\left\{\begin{array}{c}
\mathcal{D}_{t}^{\alpha} q_{k}(x, t)=D \triangle q_{k}(x, t)+A(t) q_{k}(x, t), \\
+B(t) u_{k}(x, t) \\
y_{k}(x, t)=C(t) q_{k}(x, t)+G(t) u_{k}(x, t)
\end{array}\right.
$$

where subscript $k$ denotes the iterative number of the process; $x$ and $t$ respectively denote space and time variables, $(x, t) \in \Omega \times[0, T] ; \Omega$ is a bounded open subset with smooth boundary $\partial \Omega$; $q_{k}(\cdot, \cdot) \in \mathbb{R}^{n}, u_{k}(\cdot, \cdot) \in \mathbb{R}^{u}, y_{k}(\cdot, \cdot) \in \mathbb{R}^{y}$ are the state vector, input vector and output vector of the system, respectively. The time-varying matrices $A(t), B(t), C(t), G(t)$ with appropriate dimensions are assumed to be bounded and square integrable on $[0, T]$. $D$ is a bounded positive constant diagonal matrix, i.e., $D=\operatorname{diag}\left\{d_{1}, d_{2}, \cdots, d_{n}\right\} .0<p_{i} \leq d_{i}<\infty(i=1,2, \cdots, n)$, and $p_{i}$ are known, and $\Delta=\sum_{j=1}^{m} \frac{\partial^{2}}{\partial x_{j}^{2}}$ is a Laplace operator on $\Omega$. 
Remark 1. When the fractional order $\alpha=1$, the fractional order distributed parameter System (1) is reduced to an integral order one, which has been widely investigated in [11,16-19].

The corresponding initial and boundary conditions of System (1) are:

$$
\begin{aligned}
& \alpha q_{k}(x, t)+\beta \frac{\partial q_{k}(x, t)}{\partial v}=0 \\
& q_{k}(x, 0)=q_{k 0}(x), x \in \Omega .
\end{aligned}
$$

where $(x, t) \in \partial \Omega \times[0, T], \frac{\partial}{\partial \nu}$ is the unit outward normal derivative on $\partial \Omega$ and $\alpha$ and $\beta$ are known constant diagonal matrices, satisfying:

$$
\begin{aligned}
& \alpha=\operatorname{diag}\left\{\alpha_{1}, \alpha_{2}, \cdots, \alpha_{n}\right\}, \\
& \beta=\operatorname{diag}\left\{\beta_{1}, \beta_{2}, \cdots, \beta_{n}\right\}, \\
& \alpha_{i}, \beta_{i} \geq 0, i=1,2, \cdots, n .
\end{aligned}
$$

The design objective in this paper is to find an iterative learning control law to generate the control input $u_{k}(x, t)$ such that the system output $y_{k}(x, t)$ tracks the desired output trajectory $y_{d}(x, t)$ as accurately as possible when $k$ goes to infinity for all $(x, t) \in \Omega \times[0, T]$.

To this end, the updating law considered in this manuscript is a second order P-type ILC updating law given by:

$$
\left\{\begin{aligned}
u_{k+1}(x, t) & =\gamma u_{k}(x, t)+(1-\gamma) u_{k-1}(x, t) \\
& +\Lambda e_{k}(x, t)+\Gamma e_{k+1}(x, t) \\
q_{k+1}(x, 0) & =q_{k}(x, 0)+L e_{k+1}(x, 0)
\end{aligned}\right.
$$

where $e_{k}(x, t)=y_{d}(x, t)-y_{k}(x, t)$ denotes the tracking error, $u_{0}(x, t), u_{1}(x, t)$ are two initial inputs, $0<\gamma<1$ is a constant and $\Lambda, \Gamma$ and $L$ are unknown parameters to be determined.

Remark 2. The identical initial condition is commonly used in ILC. As pointed out in [37], it will limit the application of ILC. Many modified strategies have been used to avoid this restriction. Borrowed from [37,38], we designed a P-type ILC updating law (7) with initial state learning where the strict identical initial condition is not required.

Throughout, this paper, $\mathbb{R}^{n}$ denotes an $n$-dimensional Euclidean space, and I means an identity matrix of appropriate order. For the $n$-dimensional vector $W=\left(w_{1}, w_{2}, \cdots, w_{n}\right)^{T}$, its two-norm for the $n$-dimensional vector $w=\left(w_{1}, w_{2}, \cdots, w_{n}\right)$ is defined as $\|w\|=\sqrt{\sum_{i=1}^{n} w_{i}^{2}}$, and the spectrum norm of the $n \times n$ order square matrix $A$ is $\|A\|=\sqrt{\lambda_{\max }\left(A^{T} A\right)}$, where $\lambda_{\text {max }}$ represents the maximum eigenvalue. $\rho(A)$ denotes the spectral radius of matrix $A$. Let $L^{2}(\Omega)$ be the Hilbert space. If $Q_{i} \in L^{2}(\Omega)(i=1,2, \cdots, n)$, we define $Q=\left(Q_{1}, Q_{2}, \cdots, Q_{n}\right) \in \mathbf{R}^{n} \cap L^{2}(\Omega)$, then $\|Q\|_{L^{2}}=\left\{\int_{\Omega}\left(Q(x)^{T} Q(x)\right) d x\right\}^{\frac{1}{2}}$.

For the function $f(x, t): \Omega \times[0, T] \rightarrow \mathbf{R}^{n}, f(x, t) \in \mathbf{R}^{n} \cap L^{2}(\Omega), t \in[0, T]$, we define the norm of $\left(L_{2}, \lambda\right)$ as:

$$
\|f\|_{\left(L_{2}, \lambda\right)}=\sup _{t \in[0, T]}\left\{e^{-\lambda t}\|f\|_{L^{2}}^{2}\right\}, \lambda>0 .
$$

For the function sequence $f_{k}(x, t): \Omega \times[0, T] \rightarrow \mathbf{R}^{n}, k \in\{1,2, \cdots\}$, the norm of $\left(L_{2}, \lambda, \xi\right)$ is defined as:

$$
\left\|f_{k}\right\|_{\left(L_{2}, \lambda, \xi\right)}=\sup _{t \in[0, T]}\left\{e^{-\lambda t}\left\|f_{k}\right\|_{L^{2} \xi^{k}}\right\}, \lambda>0 .
$$




\section{Convergence Analysis for Second Order P-Type ILC}

To obtain the main results, some useful lemmas are introduced.

Lemma 5 (Greens formula [39]). Let $u, v \in c^{2}(\Omega)$, then:

$$
\int_{\Omega} \nabla u \cdot \nabla v d x=-\int_{\Omega} u \triangle v d x+\int_{\partial \Omega} \frac{\partial v}{\partial v} u d s,
$$

where $\frac{\partial}{\partial v}$ is the unit outward normal derivative on $\partial \Omega$.

For fractional order linear distribute parameter System (1) with the P-type ILC updating law (7), we first give the following lemmas.

Lemma 6. Let $\delta u_{k}(x, t)=u_{k}(x, t)-u_{k-1}(x, t), \delta q_{k}(x, t)=q_{k}(x, t)-q_{k-1}(x, t)$, and:

$$
\begin{aligned}
& Q_{k}(x, t)=\left[\begin{array}{c}
e_{k}(x, t) \\
\delta u_{k}(x, t)
\end{array}\right], \\
& \Pi=\left(\begin{array}{cc}
G_{11} & G_{12} \\
\Lambda+\Gamma G_{11} & (\gamma-1) I+\Gamma G_{12}
\end{array}\right), \\
& F_{k}(x, t)=\left[\begin{array}{c}
F_{1} \delta q_{k+1}(x, t) \\
\Gamma F_{1} \delta q_{k+1}(x, t)
\end{array}\right],
\end{aligned}
$$

where:

$$
\begin{aligned}
& G_{11}=(I+G(t) \Gamma)^{-1}(I-G(t) \Lambda), \\
& G_{12}=(I+G(t) \Gamma)^{-1}(1-\gamma) G(t), \\
& F_{1}=-(I+G(t) \Gamma)^{-1} C(t)
\end{aligned}
$$

then

$$
Q_{k+1}(x, t)=\Pi Q_{k}(x, t)+F_{k}(x, t), \quad k \geq 1 .
$$

Proof. It follows from (7) that:

$$
\begin{aligned}
\delta u_{k+1}(x, t) & =(\gamma-1) \delta u_{k}(x, t) \\
& +\Lambda e_{k}(x, t)+\Gamma e_{k+1}(x, t) .
\end{aligned}
$$

Since $e_{k+1}(x, t)-e_{k}(x, t)=-\left(y_{k+1}(x, t)-y_{k}(x, t)\right)$, then from (1), it can be obtained that:

$$
\begin{aligned}
& e_{k+1}(x, t)-e_{k}(x, t) \\
= & -C(t) \delta q_{k+1}(x, t)-G(t) \delta u_{k+1}(x, t) .
\end{aligned}
$$

From (9) and (10), by some calculation, it can be obtained that:

$$
\left[\begin{array}{c}
e_{k+1}(x, t) \\
\delta u_{k+1}(x, t)
\end{array}\right]=\Pi\left[\begin{array}{c}
e_{k}(x, t) \\
\delta u_{k}(x, t)
\end{array}\right]+F_{k}(x, t) .
$$

The proof is complete.

Based on Lemma 6, it is not difficult to prove the following lemma. 
Lemma 7. For fractional order linear distribute parameter System (1), if:

$$
\rho(\Xi)<1
$$

where:

$$
\begin{aligned}
& \Xi=\left(\begin{array}{cc}
\Xi_{11} & \Xi_{12} \\
\Lambda+\Gamma \Xi_{11} & (\gamma-1) I+\Gamma \Xi_{12}
\end{array}\right), \\
& \Xi_{11}=[I+G(0) \Gamma]^{-1}[I-G(0) \Lambda-C(0) L], \\
& \Xi_{12}=[I+G(0) \Gamma]^{-1}(1-\gamma) G(0),
\end{aligned}
$$

then for all $x \in \Omega$, the second order P-type ILC updating law (7) guarantees that:

$$
\lim _{k \rightarrow \infty}\left\|Q_{k}(x, 0)\right\|_{L^{2}}^{2}=0 .
$$

Proof. According to Lemma 6, we have:

$$
\left[\begin{array}{c}
e_{k+1}(x, 0) \\
\delta u_{k+1}(x, 0)
\end{array}\right]=\bar{\Pi}\left[\begin{array}{c}
e_{k}(x, 0) \\
\delta u_{k}(x, 0)
\end{array}\right]+\bar{F}_{k}(x, 0)
$$

where:

$$
\begin{aligned}
& \bar{\Pi}=\left(\begin{array}{cc}
\bar{G}_{11} & \bar{G}_{12} \\
\Lambda+\Gamma \bar{G}_{11} & (\gamma-1) I+\Gamma \bar{G}_{12}
\end{array}\right), \\
& \bar{F}_{k}(x, 0)=\left[\begin{array}{c}
F_{1} \delta q_{k+1}(x, 0) \\
\Gamma F_{1} \delta q_{k+1}(x, 0)
\end{array}\right], \\
& \bar{G}_{11}=(I+G(0) \Gamma)^{-1}(I-G(0) \Lambda), \\
& \bar{G}_{12}=(I+G(0) \Gamma)^{-1}(1-\gamma) G(0), \\
& \bar{F}_{1}=-(I+G(0) \Gamma)^{-1} C(0),
\end{aligned}
$$

From (7), we know that:

$$
\delta q_{k+1}(x, 0)=L e_{k}(x, 0) .
$$

Substituting (14) into (13) gives:

$$
\left[\begin{array}{c}
e_{k+1}(x, 0) \\
\delta u_{k+1}(x, 0)
\end{array}\right]=\Xi\left[\begin{array}{c}
e_{k}(x, 0) \\
\delta u_{k}(x, 0)
\end{array}\right] .
$$

Therefore, according to the contracting mapping principle, we have:

$$
\lim _{k \rightarrow \infty}\left\|Q_{k}(x, 0)\right\|_{L^{2}}^{2}=0 .
$$

The proof is complete.

From Lemma 6, it can be see that the design problem of a second order P-type ILC updating law (7) for fractional order linear distributed parameter System (1) is equivalent to the stability problem of the discrete System (8). 
Lemma 8. Denote that:

$$
\begin{aligned}
M_{1} & =\left\|F_{1}\right\|+\left\|\Gamma F_{1}\right\|, M_{2}=|\gamma-1|+\|\Lambda\| \\
M_{3} & =\left\|G_{11}\right\|+\left\|G_{12}\right\|, \\
C_{1} & =\|B(t)\| \cdot\left(M_{2}+M_{3}\|\Gamma\|\right) \\
C_{2} & =\|B(t)\| \cdot\|\Gamma\| \cdot\left\|F_{1}\right\|, h=2\|A(t)\|+\|B(t)\| .
\end{aligned}
$$

If an appropriate constant $\lambda$ is chosen such that $\lambda^{\alpha}>E_{\alpha}\left(h T^{\alpha}\right) C_{2}$, then:

$$
\begin{aligned}
& \left\|F_{k}(x, t)\right\|_{\left(L^{2}, \lambda, \xi\right)} \\
\leq & \frac{\lambda^{\alpha} E_{\alpha}\left(h T^{\alpha}\right) M_{1}}{\lambda^{\alpha}-E_{\alpha}\left(h T^{\alpha}\right) C_{2}}\left\|L e_{k}(x, 0)\right\|_{L^{2}}^{2} \\
+ & \frac{E_{\alpha}\left(h T^{\alpha}\right) C_{1} M_{1}}{\lambda^{\alpha}-E_{\alpha}\left(h T^{\alpha}\right) C_{2}} \cdot\left\|Q_{k}(x, t)\right\|_{\left(L^{2}, \lambda, \xi\right)} .
\end{aligned}
$$

Proof. It follows the definition of $F_{k}(x, t)$ and the assumptions that:

$$
\begin{aligned}
& \left\|F_{k}(x, t)\right\|_{L^{2}} \\
\leq & \left(\left\|F_{1}\right\|+\left\|\Gamma F_{1}\right\|\right) \cdot\left\|\delta q_{k+1}(x, t)\right\|_{L^{2}} \\
= & M_{1}\left\|\delta q_{k+1}(x, t)\right\|_{L^{2}} .
\end{aligned}
$$

From (1), we have:

$$
\begin{aligned}
\mathcal{D}_{t}^{\alpha} \delta q_{k}(x, t) & =D \triangle \delta q_{k}(x, t)+A(t) \delta q_{k}(x, t) \\
& +B(t) \delta u_{k}(x, t) .
\end{aligned}
$$

Note that:

$$
\begin{aligned}
& \mathcal{D}_{t}^{\alpha}\left(\left\|\delta q_{k}(x, t)\right\|_{L^{2}}^{2}\right) \\
= & \int_{\Omega} \mathcal{D}_{t}^{\alpha}\left(\delta q_{k}^{T}(x, t) \delta q_{k}(x, t)\right) d x \\
\leq & 2 \int_{\Omega} \delta q_{k}^{T}(x, t) \mathcal{D}_{t}^{\alpha} \delta q_{k}(x, t) d x \\
= & 2 \int_{\Omega} \delta q_{k}^{T}(x, t) D \triangle \delta q_{k}(x, t) d x \\
+ & \int_{\Omega} \delta q_{k}^{T}(x, t)\left(A^{T}(t)+A(t)\right) \delta q_{k}(x, t) d x \\
+ & 2 \int_{\Omega} \delta q_{k}^{T}(x, t) B(t) \delta u_{k}(x, t) d x \\
:= & I_{1}+I_{2}+I_{3} .
\end{aligned}
$$

Then, using the Green formula and the boundary condition (2) to $I_{1}$, we have:

$$
\begin{aligned}
I_{1} & =2 \sum_{i=1}^{n} d_{i} \int_{\Omega} \delta q_{k i}^{T}(x, t) \triangle \delta q_{k i}(x, t) d x \\
& =2 \sum_{i=1}^{n} d_{i} \int_{\Omega} \delta q_{k i}^{T}(x, t) \frac{\partial \delta q_{k i}(x, t)}{\partial v} d x \\
& -2 \sum_{i=1}^{n} d_{i} \int_{\Omega} \nabla q_{k i}^{T}(x, t) \nabla q_{k i}(x, t) d x \\
& =2 \sum_{i=1}^{n} d_{i} \int_{\Omega} \delta q_{k i}^{T}(x, t)\left(-\frac{\alpha_{i}}{\beta_{i}} \delta q_{k i}(x, t)\right) d x \\
& -2 \sum_{i=1}^{n} d_{i} \int_{\Omega} \nabla q_{k i}^{T}(x, t) \nabla q_{k i}(x, t) d x \\
& \leq 0 .
\end{aligned}
$$


Clearly,

$$
\begin{aligned}
I_{2} & \leq 2\|A(t)\| \int_{\Omega} \delta q_{k}^{T}(x, t) \delta q_{k}(x, t) d x \\
& =2\|A(t)\| \cdot\left\|\delta q_{k}(x, t)\right\|_{L^{2}}^{2} .
\end{aligned}
$$

Using the Hölder inequality of $I_{3}$, we get:

$$
\begin{aligned}
I_{3} & \leq\|B(t)\| \delta q_{k}(x, t) \|_{L^{2}}^{2} \\
& +\|B(t)\| \delta u_{k}(x, t) \|_{L^{2}}^{2}
\end{aligned}
$$

Therefore, from (20) to (23), we obtain:

$$
\begin{aligned}
& \mathcal{D}_{t}^{\alpha}\left(\left\|\delta q_{k}(x, t)\right\|_{L^{2}}^{2}\right) \\
\leq & (2\|A(t)\|+\|B(t)\|) \delta q_{k}(x, t) \|_{L^{2}}^{2} \\
+ & \|B(t)\| \delta u_{k}(x, t) \|_{L^{2}}^{2} .
\end{aligned}
$$

Integrating both sides of (24) above $t$, we can get:

$$
\begin{aligned}
& \left\|\delta q_{k}(x, t)\right\|_{L^{2}}^{2} \\
\leq & \left\|\delta q_{k}(x, 0)\right\|_{L^{2}}^{2} \\
+ & \frac{g}{\Gamma(\alpha)} \int_{0}^{t}(t-s)^{\alpha-1}\left\|\delta u_{k}(x, s)\right\|_{L^{2}}^{2} d s \\
+ & \frac{h}{\Gamma(\alpha)} \int_{0}^{t}(t-s)^{\alpha-1}\left\|\delta q_{k}(x, s)\right\|_{L^{2}}^{2} d s, \\
\leq & \left\|\delta q_{k}(x, 0)\right\|_{L^{2}}^{2} \\
+ & \frac{g}{\Gamma(\alpha)} \int_{0}^{t}(t-s)^{\alpha-1} e^{\lambda s} d s\left\|\delta u_{k}(x, s)\right\|_{\left(L^{2}, \lambda\right)}^{2} \\
+ & \frac{h}{\Gamma(\alpha)} \int_{0}^{t}(t-s)^{\alpha-1}\left\|\delta q_{k}(x, s)\right\|_{L^{2}}^{2} d s
\end{aligned}
$$

where $g=\|B(t)\|, h=2\|A(t)\|+\|B(t)\|$. Following from Lemma 3 and the definition of the Mittag-Leffler function that for $\lambda>0$, we have:

$$
\frac{d t^{\alpha} E_{1,1+\alpha}(\lambda t)}{d t}=t^{\alpha-1} E_{1, \alpha}(\lambda t)>0 .
$$

As a result,

$$
\frac{g}{\Gamma(\alpha)} \int_{0}^{t}(t-s)^{\alpha-1} e^{\lambda s} d s=g t^{\alpha} E_{1,1+\alpha}(\lambda t)
$$

is an increasing function.

Using the generalized Gronwall inequality of Inequality (25), it results:

$$
\begin{aligned}
& \left\|\delta q_{k}(x, t)\right\|_{L^{2}}^{2} \\
\leq & \left(\left\|\delta q_{k}(x, 0)\right\|_{L^{2}}^{2}\right. \\
+ & \left.\frac{g}{\Gamma(\alpha)} \int_{0}^{t}(t-s)^{\alpha-1} e^{\lambda s} d s\left\|\delta u_{k}(x, s)\right\|_{\left(L^{2}, \lambda\right)}^{2}\right) \\
\times & E_{\alpha}\left(h t^{\alpha}\right) .
\end{aligned}
$$


Furthermore, it can be easily proven that:

$$
\begin{aligned}
& \int_{0}^{t}(t-s)^{\alpha-1} e^{\lambda s} d s \\
= & \int_{0}^{t} w^{\alpha-1} e^{\lambda(t-w)} d w \\
= & e^{\lambda t} \int_{0}^{t} w^{\alpha-1} e^{-\lambda w} d w \\
= & \frac{e^{\lambda t}}{\lambda^{\alpha}} \int_{0}^{\lambda t} s^{\alpha-1} e^{-s} d s<\frac{e^{\lambda t}}{\lambda^{\alpha}} \Gamma(\alpha) .
\end{aligned}
$$

Substituting it into Inequality (26), one has:

$$
\begin{aligned}
& \left\|\delta q_{k}(x, t)\right\|_{L^{2}}^{2} \\
\leq & \left(\left\|\delta q_{k}(x, 0)\right\|_{L^{2}}^{2}\right. \\
+ & \left.\frac{g e^{\lambda t}}{\lambda^{\alpha}}\left\|\delta u_{k}(x, s)\right\|_{\left(L^{2}, \lambda\right)}^{2}\right) \times E_{\alpha}\left(h t^{\alpha}\right) .
\end{aligned}
$$

Multiplying both sides of the above inequality by $e^{-\lambda t}$, one has:

$$
\begin{aligned}
& \left\|\delta q_{k}(x, t)\right\|_{L^{2}}^{2} e^{-\lambda t} \\
\leq & e^{-\lambda t}\left\|\delta q_{k}(x, 0)\right\|_{L^{2}}^{2} \times E_{\alpha}\left(h T^{\alpha}\right) \\
+ & \frac{g}{\lambda^{\alpha}}\left\|\delta u_{k}(x, s)\right\|_{\left(L^{2}, \lambda\right)}^{2} \times E_{\alpha}\left(h T^{\alpha}\right) \\
\leq & \left\|\delta q_{k}(x, 0)\right\|_{L^{2}}^{2} \times E_{\alpha}\left(h T^{\alpha}\right) \\
+ & \frac{g}{\lambda^{\alpha}}\left\|\delta u_{k}(x, s)\right\|_{\left(L^{2}, \lambda\right)}^{2} \times E_{\alpha}\left(h T^{\alpha}\right) .
\end{aligned}
$$

It follows from (7), and we have $\left\|\delta q_{k}(x, 0)\right\|_{L^{2}}^{2} \leq\left\|L e_{k}(x, 0)\right\|_{L^{2}}^{2}$. By substituting it into (29), we can obtain:

$$
\begin{aligned}
& \left\|\delta q_{k+1}(x, t)\right\|_{\left(L^{2}, \lambda\right)} \\
\leq & E_{\alpha}\left(h T^{\alpha}\right) \times\left\|L e_{k}(x, 0)\right\|_{L^{2}}^{2} \\
+ & \frac{g}{\lambda^{\alpha}} \times E_{\alpha}\left(h T^{\alpha}\right)\left\|\delta u_{k+1}(x, t)\right\|_{\left(L^{2}, \lambda\right)} .
\end{aligned}
$$

On the other hand, it follows from (9) that:

$$
\begin{aligned}
& \left\|\delta u_{k+}(x, t)\right\|_{\left(L^{2}, \lambda\right)} \\
\leq & \left|(\gamma-1)\left\|\left.\Delta u_{k}(x, t)\right|_{\left(L^{2}, \lambda\right)}+\right\| \Lambda\|\| e_{k}(x, t) \|_{\left(L^{2}, \lambda\right)}\right. \\
+ & \|\Gamma\| \cdot\left\|e_{k+1}(x, t)\right\|_{\left(L^{2}, \lambda\right)} \\
\leq & M_{2}\left\|Q_{k}(x, t)\right\|_{\left(L^{2}, \lambda\right)} \\
+ & \|\Gamma\| \cdot\left\|e_{k+1}(x, t)\right\|_{\left(L^{2}, \lambda\right)},
\end{aligned}
$$

where $M_{2}=|(\gamma-1)|+\|\Lambda\|$.

According to (8), we have:

$$
\begin{aligned}
& \left\|e_{k+1}(x, t)\right\|_{\left(L^{2}, \lambda\right)} \\
\leq & \left\|G_{11}\right\|\left|\Delta e_{k}(x, t)\right|_{\left(L^{2}, \lambda\right)}+\left\|G_{12}\right\|\left\|\delta u_{k}(x, t)\right\|_{\left(L^{2}, \lambda\right)} \\
+ & \left\|F_{1}\right\| \cdot\left\|\delta q_{k+1}(x, t)\right\|_{\left(L^{2}, \lambda\right)} \\
\leq & M_{3}\left\|Q_{k}(x, t)\right\|_{\left(L^{2}, \lambda\right)} \\
+ & \left\|F_{1}\right\| \cdot\left\|\delta q_{k+1}(x, t)\right\|_{\left(L^{2}, \lambda\right)},
\end{aligned}
$$


where $M_{3}=\left\|G_{11}\right\|+\left\|G_{12}\right\|$. As a result, we obtain from (30) to (32) that:

$$
\begin{aligned}
& \left\|\delta q_{k+1}(x, t)\right\|_{\left(L^{2}, \lambda\right)} \\
\leq & E_{\alpha}\left(h T^{\alpha}\right) \times\left\|L e_{k}(x, 0)\right\|_{L^{2}}^{2} \\
+ & \frac{g}{\lambda^{\alpha}} E_{\alpha}\left(h T^{\alpha}\right)\left(M_{2}+M_{3}\|\Gamma\|\right) \cdot\left\|Q_{k}(x, t)\right\|_{\left(L^{2}, \lambda\right)} \\
+ & E_{\alpha}\left(h T^{\alpha}\right) \frac{g\|\Gamma\| \cdot\left\|F_{1}\right\|}{\lambda^{\alpha}}\left\|\delta q_{k+1}(x, t)\right\|_{\left(L^{2}, \lambda\right)} .
\end{aligned}
$$

Thus, by defining $g\left(M_{2}+M_{3}\|\Gamma\|\right)=C_{1}, g\|\Gamma\| \cdot\left\|F_{1}\right\|=C_{2}$ and selecting a sufficiently large $\lambda$ such that $\lambda^{\alpha}>E_{\alpha}\left(h T^{\alpha}\right) C_{2}$, we can get:

$$
\begin{aligned}
& \left\|\delta q_{k+1}(x, t)\right\|_{\left(L^{2}, \lambda\right)} \\
\leq & \frac{\lambda^{\alpha} E_{\alpha}\left(h T^{\alpha}\right)}{\lambda^{\alpha}-E_{\alpha}\left(h T^{\alpha}\right) C_{2}}\left\|L e_{k}(x, 0)\right\|_{L^{2}}^{2} \\
+ & \frac{E_{\alpha}\left(h T^{\alpha}\right) C_{1}}{\lambda^{\alpha}-E_{\alpha}\left(h T^{\alpha}\right) C_{2}} \cdot\left\|Q_{k}(x, t)\right\|_{\left(L^{2}, \lambda\right)} .
\end{aligned}
$$

By applying the $\left(L^{2}, \lambda\right)$-norm and substituting the above equality into (18), we can obtain:

$$
\begin{aligned}
& \left\|F_{k}(x, t)\right\|_{\left(L^{2}, \lambda\right)} \\
\leq & \frac{\lambda^{\alpha} E_{\alpha}\left(h T^{\alpha}\right) M_{1}}{\lambda^{\alpha}-E_{\alpha}\left(h T^{\alpha}\right) C_{2}}\left\|L e_{k}(x, 0)\right\|_{L^{2}}^{2} \\
+ & \frac{E_{\alpha}\left(h T^{\alpha}\right) C_{1} M_{1}}{\lambda^{\alpha}-E_{\alpha}\left(h T^{\alpha}\right) C_{2}} \cdot\left\|Q_{k}(x, t)\right\|_{\left(L^{2}, \lambda\right)} .
\end{aligned}
$$

Furthermore, applying the $\left(L^{2}, \lambda, \xi\right)$-norm to Inequality (35), it yields (17), which completes the proof.

In the following, based on Lemmas 6 to 8 , a convergent condition for both tracking errors and control input is derived for fractional order linear distributed parameter System (1).

Theorem 1. For fractional order linear distributed parameter System (1) and a given reference $y_{d}(x, t)$, suppose that the condition in Lemma 7 is satisfied. If:

$$
\rho(\Pi) \leq \rho_{0}<1,
$$

where $\Pi$ is the same as in Lemma 6 , then for all $(x, t) \in \Omega \times[0, T]$, the second order P-type ILC updating law (7) guarantees that:

$$
\lim _{k \rightarrow \infty}\left\|e_{k}(x, t)\right\|_{L^{2}}=0
$$

Proof. It follows from (8) for $k>1$ that:

$$
Q_{k}(x, t)=\Pi^{k-1} Q_{1}(x, t)+\sum_{i=1}^{k-1} \Pi^{k-i-1} F_{i}(x, t) .
$$

Therefore,

$$
\begin{aligned}
& \left\|Q_{k}(x, t)\right\|_{L^{2}} \\
\leq & \{\rho(\Pi)\}^{k-1}\left\|Q_{1}(x, t)\right\|_{L^{2}} \\
+ & \sum_{i=1}^{k-1}\{\rho(\Pi)\}^{k-i-1}\left\|F_{i}(x, t)\right\|_{L^{2}} .
\end{aligned}
$$


Noting that $\rho(\Pi) \leq \rho_{0}<1$ by assumption, then there exists a constant $\xi>1, \rho_{0} \xi<1$, and a sufficiently large $\lambda$ such that $\lambda^{\alpha}>E_{\alpha}\left(h T^{\alpha}\right) C_{2}$, and:

$$
0<\gamma=\frac{\xi}{1-\rho_{0} \xi} \cdot \frac{E_{\alpha}\left(h T^{\alpha}\right) C_{1} M_{1}}{\lambda^{\alpha}-E_{\alpha}\left(h T^{\alpha}\right) C_{2}}<1
$$

For the above $\lambda$ and $\xi$, multiplying both sides of Inequality (39) by $e^{-\lambda t} \xi^{k}$ and taking the $\left(L^{2}, \lambda, \xi\right)$-norm, it yields:

$$
\begin{aligned}
& \left(\left\|Q_{k}(x, t)\right\|_{L^{2}} \xi^{k}\right) e^{-\lambda t} \\
\leq & \rho_{0}^{k-1} \xi^{k}\left\|Q_{1}(x, t)\right\|_{L^{2}} e^{-\lambda t} \\
+ & \sum_{i=1}^{k-1} \rho_{0}^{k-i-1} \xi^{k}\left\|F_{i}(t)\right\|_{L^{2}} e^{-\lambda t} \\
\leq & \left.\left(\rho_{0} \xi\right)^{k} \rho_{0}^{-1} \| Q_{1}(x, t)\right) \|_{L^{2}} e^{-\lambda t} \\
+ & \sum_{i=1}^{k-1}\left(\rho_{0} \xi\right)^{k-i-1} \xi\left(\left\|F_{i}(t)\right\|_{L^{2}} \xi^{i}\right) e^{-\lambda t} \\
\leq & \rho_{0}^{-1}\left\|Q_{1}(x, t)\right\|_{\left(L^{2}, \lambda\right)} \\
+ & \frac{\xi}{1-\rho_{0} \xi} \sup _{1 \leq i \leq k}\left\|F_{i}(t)\right\|_{\left(L^{2}, \lambda, \xi\right)}
\end{aligned}
$$

It follows from (17) and (41), and we have:

$$
\begin{aligned}
& \left\|Q_{k}(x, t)\right\|_{\left(L^{2}, \lambda, \xi\right)} \\
\leq & \rho_{0}^{-1}\left\|Q_{1}(x, t)\right\|_{\left(L^{2}, \lambda\right)} \\
+ & \gamma \sup _{1 \leq i \leq k}\left\|Q_{i}(x, t)\right\|_{\left(L^{2}, \lambda, \xi\right)} \\
+ & \frac{\xi}{1-\rho_{0 \zeta}} \cdot \frac{\lambda^{\alpha} E_{\alpha}\left(h T^{\alpha}\right) M_{1}}{\lambda^{\alpha}-E_{\alpha}\left(h T^{\alpha}\right) C_{2}}\|L\| E_{0},
\end{aligned}
$$

where $E_{0}=\sup _{1 \leq i \leq k-1}\left\|e_{k}(x, 0)\right\|_{L^{2}}^{2}$. Therefore,

$$
\begin{aligned}
& \sup _{1 \leq i \leq k}\left\|Q_{i}(x, t)\right\|_{\left(L^{2}, \lambda, \xi\right)} \\
\leq & \rho_{0}^{-1}\left\|Q_{1}(x, t)\right\|_{\left(L^{2}, \lambda\right)} \\
+ & \gamma \sup _{1 \leq i \leq k}\left\|Q_{i}(x, t)\right\|_{\left(L^{2}, \lambda, \xi\right)} \\
+ & \frac{\xi}{1-\rho_{0} \xi} \cdot \frac{\lambda^{\alpha} E_{\alpha}\left(h T^{\alpha}\right) M_{1}}{\lambda^{\alpha}-E_{\alpha}\left(h T^{\alpha}\right) C_{2}}\|L\| E_{0} .
\end{aligned}
$$

It can be obtained that,

$$
\begin{aligned}
& \sup _{1 \leq i \leq k}\left\|Q_{i}(x, t)\right\|_{\left(L^{2}, \lambda, \xi\right)} \\
\leq & \frac{\rho_{0}^{-1}}{1-\gamma}\left\|Q_{1}(x, t)\right\|_{\left(L^{2}, \lambda\right)} \\
+ & \frac{\xi\|L\| E_{0}}{\left(1-\rho_{0} \xi\right)(1-\gamma)} \cdot \frac{\lambda^{\alpha} E_{\alpha}\left(h T^{\alpha}\right) M_{1}}{\lambda^{\alpha}-E_{\alpha}\left(h T^{\alpha}\right) C_{2}} .
\end{aligned}
$$

Note that:

$$
\begin{aligned}
& \left\|Q_{k}(x, t)\right\|_{L^{2}} \\
= & \xi^{-k} e^{\lambda t}\left(\left\|Q_{k}(x, t)\right\|_{L^{2}} \xi^{k}\right) e^{-\lambda t} \\
\leq & \xi^{-k} e^{\lambda t} \sup _{1 \leq i \leq k}\left\|Q_{i}(x, t)\right\|_{\left(L^{2}, \lambda, \xi\right)} .
\end{aligned}
$$


Consequently, one obtains from (44) and (45) that:

$$
\begin{aligned}
& \left\|Q_{k}(x, t)\right\|_{L^{2}} \\
\leq & \frac{\rho_{0}^{-1} e^{\lambda T}}{(1-\gamma) \xi^{k}}\left\|Q_{1}(x, t)\right\|_{\left(L^{2}, \lambda\right)} \\
+ & \frac{\xi\|L\| E_{0} e^{\lambda T}}{\xi^{k}\left(1-\rho_{0} \xi\right)(1-\gamma)} \\
\times \quad & \frac{\lambda^{\alpha} E_{\alpha}\left(h T^{\alpha}\right) M_{1}}{\lambda^{\alpha}-E_{\alpha}\left(h T^{\alpha}\right) C_{2}} .
\end{aligned}
$$

Note that the condition in Lemma 7 is satisfied. Theretofore, $E_{0}$ is convergent and bounded. Besides, $r<1, \xi>1$, it follows from (46) that:

$$
\lim _{k \rightarrow \infty}\left\|Q_{k}(x, t)\right\|_{L^{2}}=0 .
$$

Thus, for all $(x, t) \in \Omega \times[0, T], k=1,2, \ldots$, we have:

$$
\lim _{k \rightarrow \infty}\left\|e_{k}(x, t)\right\|_{L^{2}}=0 .
$$

The proof is complete.

In particular, we have the following corollary.

Corollary 1. For fractional order linear distributed parameter System (1) and a given reference $y_{d}(x, t)$, if:

$$
\rho\left\{(I+G(0) \Gamma)^{-1}(I-G(0) \Lambda-C(0) L)\right\}<1,
$$

and:

$$
\rho\left\{(I+G(t) \Gamma)^{-1}(I-G(t) \Lambda)\right\}<1,
$$

then for all $(x, t) \in \Omega \times[0, T]$, the following P-type ILC updating law:

$$
\left\{\begin{array}{l}
u_{k+1}(x, t)=u_{k}(x, t)+\Lambda e_{k}(x, t)+\Gamma e_{k+1}(x, t), \\
q_{k+1}(x, 0)=q_{k}(x, 0)+L e_{k}(x, 0),
\end{array}\right.
$$

guarantees that:

$$
\lim _{k \rightarrow \infty}\left\|e_{k}(x, t)\right\|_{L^{2}}=0 .
$$

Proof. Setting $\gamma=1$, from Theorem 1, it can be concluded that if:

$$
\rho\left(\Xi_{1}\right)<1,
$$

where:

$$
\begin{aligned}
& \Xi_{1}=\left(\begin{array}{cc}
\Xi_{11} & 0 \\
\Lambda+\Gamma \Xi_{11} & 0
\end{array}\right) \\
& \Xi_{11}=[I+G(0) \Gamma]^{-1}[I-G(0) \Lambda-C(0) L]
\end{aligned}
$$

and:

$$
\rho\left(\Pi_{1}\right)=\rho\left(\begin{array}{cc}
G_{11} & 0 \\
\Lambda+\Gamma G_{11} & 0
\end{array}\right)<1,
$$


where $G_{11}=(I+G(t) \Gamma)^{-1}(I-G(t) \Lambda)$, then the P-type ILC updating law (51) guarantees that $\lim _{k \rightarrow \infty}\left\|e_{k}(x, t)\right\|_{L^{2}}=0$.

Noting that:

$$
\operatorname{det}\left(\Xi_{1}\right)=\left|\begin{array}{cc}
\lambda I-\Xi_{11} & 0 \\
-\Lambda-\Gamma \Xi_{11} & \lambda I
\end{array}\right|,
$$

and:

$$
\operatorname{det}\left(\Pi_{1}\right)=\left|\begin{array}{cc}
\lambda I-G_{11} & 0 \\
-\Lambda-\Gamma G_{11} & \lambda I
\end{array}\right|,
$$

it follows from the definition of the spectral radius that:

$$
\rho\left(\Xi_{1}\right)=\rho\left(\Pi_{1}\right), \rho\left(\Pi_{1}\right)=\rho\left(G_{11}\right) .
$$

The proof is completed.

The following corollary is obvious.

Corollary 2. For fractional order linear distributed parameter System (1) and a given reference $y_{d}(x, t)$, if:

$$
\rho\left\{(I+G(0) \Gamma)^{-1}(I-C(0) L)\right\}<1,
$$

and:

$$
\rho\left\{(I+G(t) \Gamma)^{-1}\right\}<1,
$$

then for all $(x, t) \in \Omega \times[0, T]$, the close-loop P-type ILC updating law:

$$
\left\{\begin{array}{l}
u_{k+1}(x, t)=u_{k}(x, t)+\Gamma e_{k+1}(x, t) \\
q_{k+1}(x, 0)=q_{k}(x, 0)+L e_{k}(x, 0)
\end{array}\right.
$$

guarantees that:

$$
\lim _{k \rightarrow \infty}\left\|e_{k}(x, t)\right\|_{L^{2}}=0
$$

From the proof of Theorem 1, it is not difficult to show the following Corollary.

Corollary 3. For fractional order linear distributed parameter System (1) and a given reference $y_{d}(x, t)$, suppose that in the learning process, the system states start from the same initial value, or more generally,

$$
\begin{array}{r}
q_{k}(x, 0)=\varphi_{k}(x), x \in \Omega, k=0,1,2, \cdots, \\
\left\|\varphi_{k+1}(x)-\varphi_{k}(x)\right\|_{L^{2}}^{2} \leq l r^{k}, r \in[0,1), l>0,
\end{array}
$$

if:

$$
\rho\left\{(I+G(t) \Gamma)^{-1}\right\}<1,
$$

then for all $(x, t) \in \Omega \times[0, T]$, the close-loop P-type ILC updating law:

$$
u_{k+1}(x, t)=u_{k}(x, t)+\Gamma e_{k+1}(x, t),
$$

guarantees that:

$$
\lim _{k \rightarrow \infty}\left\|e_{k}(x, t)\right\|_{L^{2}}=0
$$


Remark 3. It should be pointed out that the derived result in Corollary 3 is still true for the integral order $(\alpha=1)$ case. Note that the convergence analysis of ILC updating law (62) for integer order linear distributed parameter System (1) has been investigated in [19], in which the convergence condition is:

$$
\left\|(I+G(t) \Gamma)^{-1}\right\|^{2} \leq \rho_{0}<\frac{1}{2} .
$$

Since $\rho\left\{(I+G(t) \Gamma)^{-1}\right\} \leq\left\|(I+G(t) \Gamma)^{-1}\right\|$, the convergence Condition (61) is less conservative than Condition (64) (see, e.g., the example in Section 4).

\section{Numerical Example}

In this section, two numerical examples are presented to demonstrate the validity of the design method.

Consider a tubular flow reactor with a cooling jacket where an irreversible exothermic zeroth order reaction takes place. The reaction rate is considered to be temperature dependent, then it varies with time and the location of the reactor. The cooling jacket and feed temperatures are chosen as the manipulated variables. The thermal spatiotemporal dynamics can be derived from the energy balance inside the reactor and can be presented as the following distributed parameter systems [40]:

$$
\begin{aligned}
\frac{\partial T}{\partial t} & =\frac{k}{\rho C_{p}} \frac{\partial^{2} T}{\partial z^{2}}-u \frac{\partial T}{\partial z} \\
& -\frac{h A_{c}}{\rho C_{p}} T+\frac{(-\Delta H)}{\rho C_{p}} r_{0} e^{\left(\frac{-E}{R T}\right)},
\end{aligned}
$$

where $T$ is the stream temperature inside the reactor, $t$ is time and $z \in[0, L]$ denotes the spatial coordinate, $L$ is the reactor length. The terms $k, \rho, C_{p}, u,(-\Delta H), r_{0}, E$ and $h$ are used for thermal conductivity, density, heat capacity, axial velocity, heat of reaction, pre-exponential reaction constant, activation energy and the heat transfer coefficient between the reactor and cooling jacket, respectively. $A_{c}$ presents the cooling jacket temperature.

In the vicinity of the steady-state working point, nonlinear Model (65) can be linearized as [41]:

$$
\frac{\partial T(z, t)}{\partial t}=\theta_{1} \frac{\partial^{2} T}{\partial z^{2}}+\theta_{2}(t) T(z, t)+\theta_{3}(t) U(z, t),
$$

where $\theta_{2}(t)$ and $\theta_{3}(t)$ vary nonlinearly with temperature during the entire heating process. Define the measured value of the thermocouple as:

$$
Y(z, t)=\theta_{4}(t) T(z, t)+\theta_{5}(t) U(z, t) .
$$

In order to simplify the example, set the above distributed parameter system with fractional order $\alpha=0.9$ and:

$$
\begin{aligned}
& \theta_{1}=I_{2}, \theta_{2}(t)=\left[\begin{array}{cc}
e^{-2 t} & 0 \\
0 & e^{-3 t}
\end{array}\right], \\
& \theta_{3}(t)=\left[\begin{array}{cc}
e^{-t} & 0 \\
0.5 & e^{-1.5 t}
\end{array}\right], \\
& \theta_{4}(t)=\left[\begin{array}{ll}
0.5 & 1 \\
0.9 & 2
\end{array}\right], \theta_{5}(t)=\left[\begin{array}{cc}
0.98 & 0 \\
1.80 & 1.5
\end{array}\right] .
\end{aligned}
$$

where $(z, t) \in[0,1] \times[0,0.8]$. 
Let the reference be $y_{1 d}=(1-\cos z) t, y_{2 d}=\sin \left(2 \pi^{2} t\right) \cos (2 \pi z)$. For the above system, we apply the following P-type ILC updating law:

$$
u_{k+1}(z, t)=u_{k}(z, t)+\Gamma e_{k+1}(z, t),
$$

where $\Gamma=\left[\begin{array}{cc}0.98 & 0 \\ 1.80 & 1.5\end{array}\right]$. The initial state value is $q_{0}(z, 0)=\sin z$, while the control input value at the beginning of learning is set to zero.

By some straightforward calculations, we can find:

$$
\left\|(I+G(t) \Gamma)^{-1}\right\|^{2}=0.81>0.50 .
$$

That is, the convergence Condition (64) is not satisfied (Theorem 1 in [19] is invalid). However,

$$
\rho\left\{(I+G(t) \Gamma)^{-1}\right\}=0.51<1 .
$$

From Corollary 3, it can be concluded that the tracking error is convergent.

To simulate the fractional order operator, we use the following expression: [42]

$$
\Delta^{\alpha} y(k)=\frac{1}{h^{\alpha}} \sum_{j=0}^{L}(-1)^{j}\left(\begin{array}{c}
\alpha \\
j
\end{array}\right) y(k-j)
$$

where $h$ represents the sampling interval and the number of samples is denoted as $k .\left(\begin{array}{c}\alpha \\ j\end{array}\right)$ is the notation of the binomial coefficients. To conserve computing resources, the length of the memory $L$ is set as a finite recording number [42], usually $L=20$. To obtain the numerical solution, we use the two order explicit center difference scheme, in which the sampling periods are taken as $\Delta z=0.1, \Delta t=0.001$.

From the viewpoint of viscoelastic theory, the initial history (terminal) should be the state over a time-interval, not a state at a given time $[43,44]$. To consider the physically consistent initial conditions, a simple solution could be obtained by the procedure proposed in $[43,45]$. To simplify this discussion, the initial time of setting the integral is zero.

Figures 1 and 2 are the desired curved surfaces. Figures 3 and 4 show the system output of the ninth iterations. It can be seen that the system output is capable of approaching the desired trajectory accurately within a few iterations. Figures 5 and 6 are the error surface when iteration number $k=9$. When the number of iterations is nine, the absolute values of the maximum tracking error are $1.376 \times 10^{-4}, 1.583 \times 10^{-2}$, respectively. Figure 7 shows the $L_{2}$-norm of the tracking errors in each iteration. Thus, Figures 1 to 7 show that the designed ILC is effective.

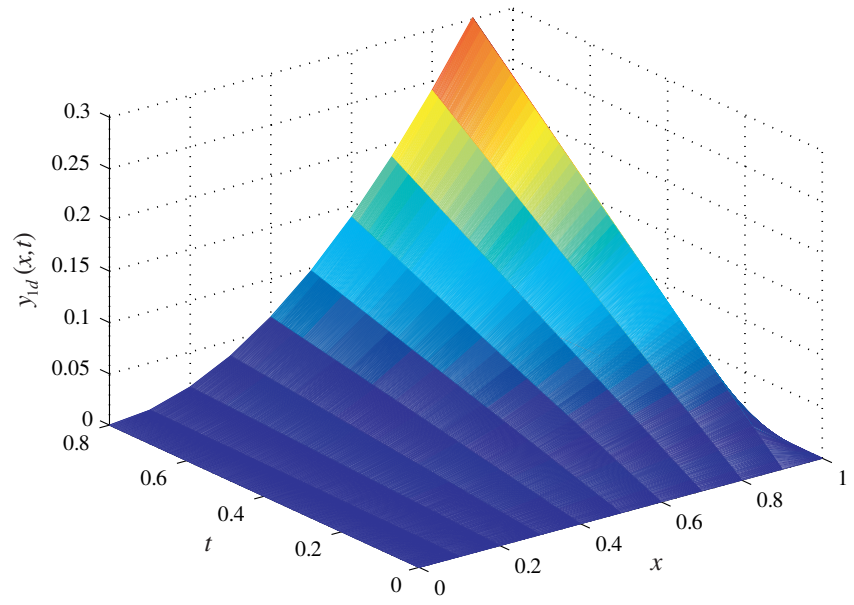

Figure 1. The desired output $y_{1 d}$. 


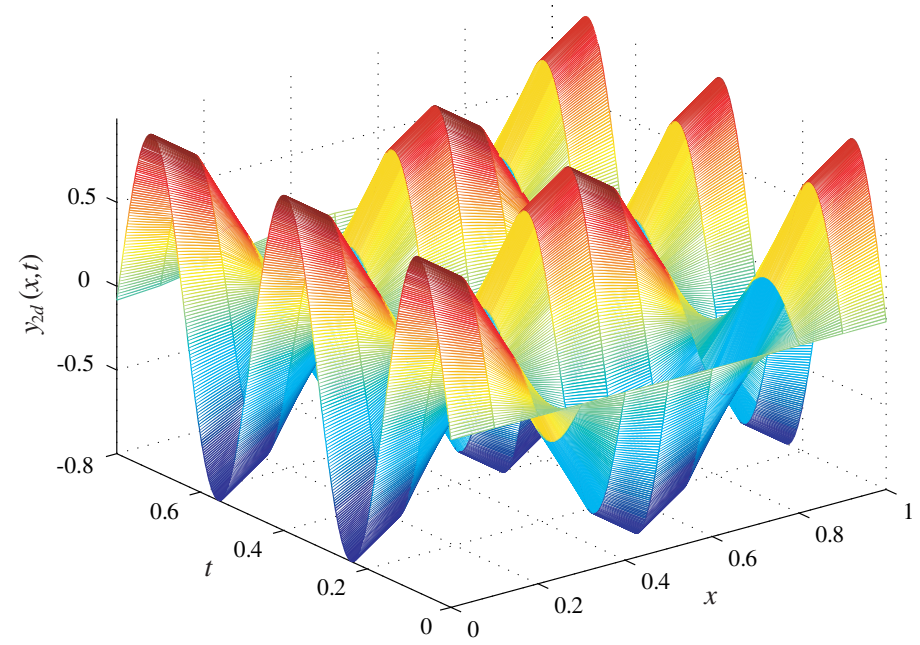

Figure 2. The desired output $y_{2 d}$.

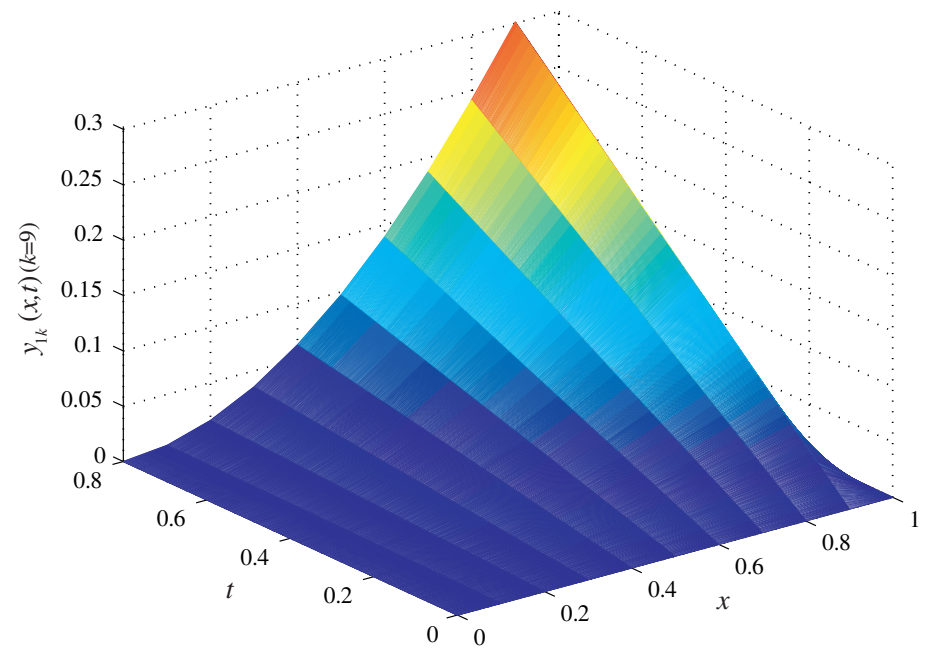

Figure 3. The ninth iterative output $y_{1 k}(k=9)$.

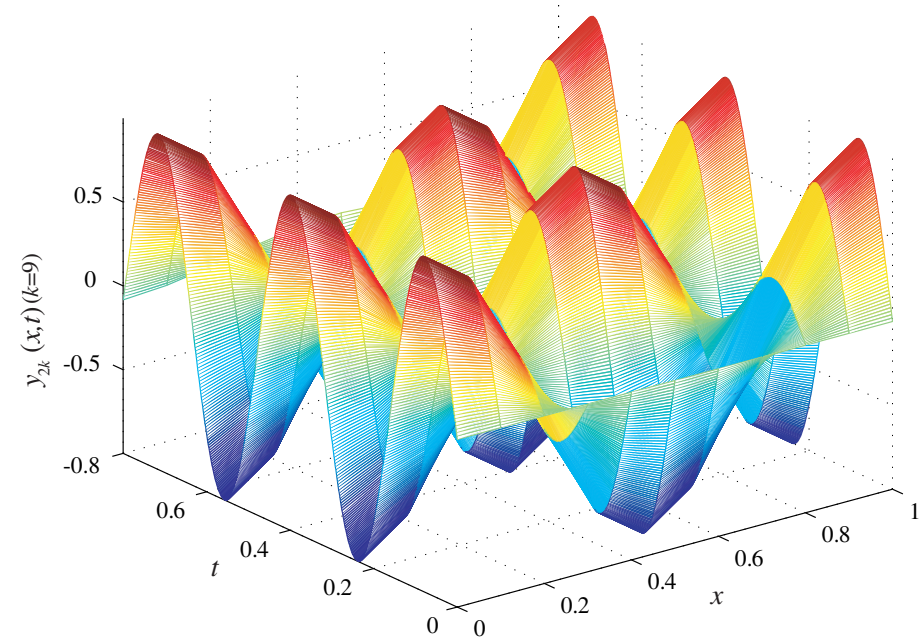

Figure 4. The ninth iterative output $y_{2 k}(k=9)$. 


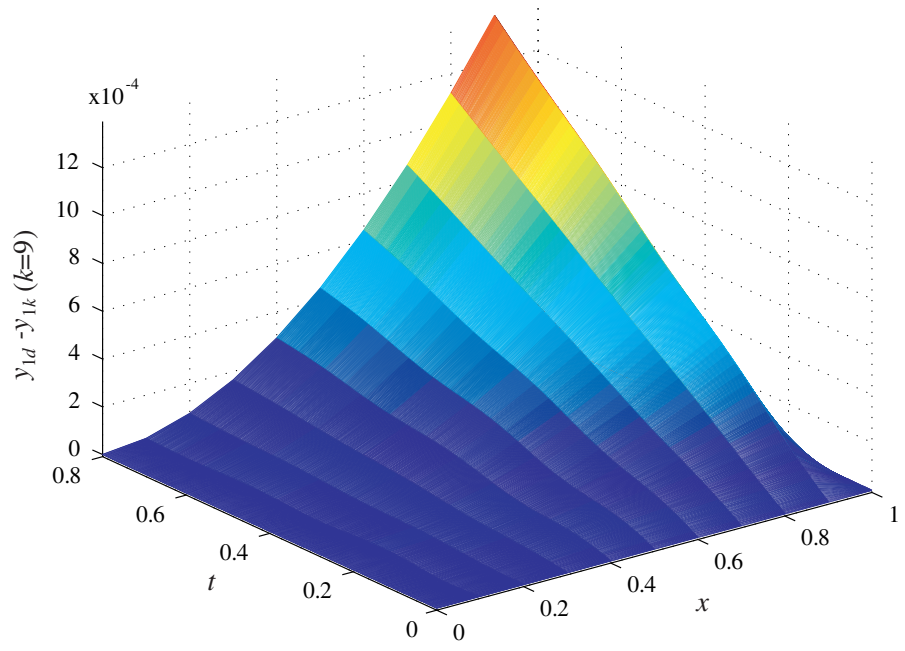

Figure 5. The error surface $y_{1 d}-y_{1 k}(k=9)$.

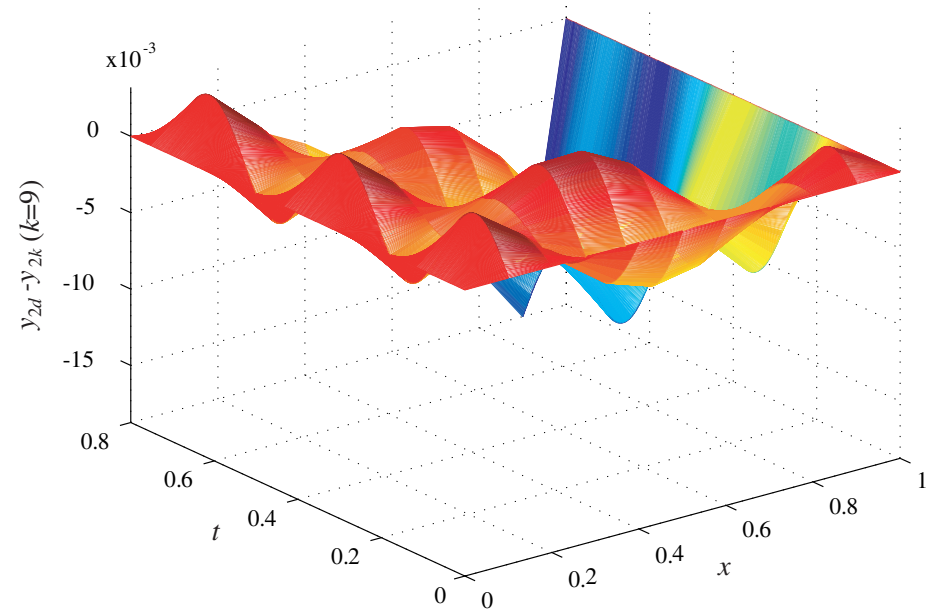

Figure 6. The error surface $y_{2 d}-y_{2 k}(k=9)$.

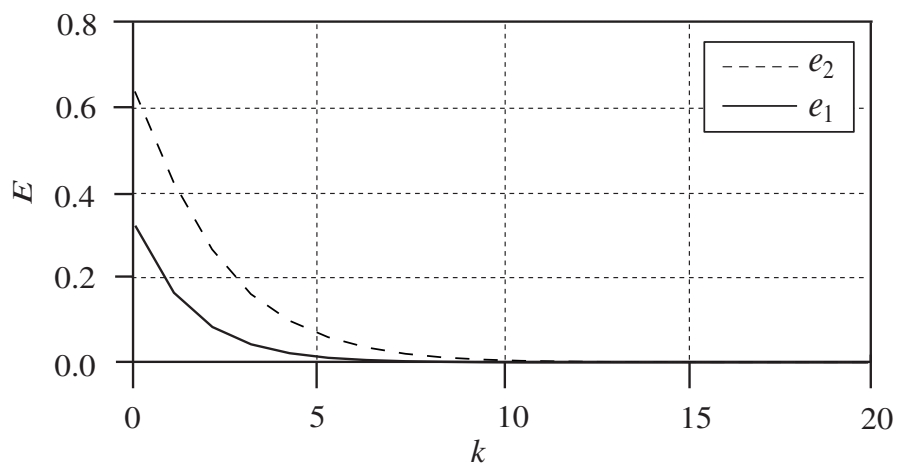

Figure 7. The iterative number-max tracking error.

Let the reference be $y_{1 d}(t)$ and the fractional order $\alpha \in\{0.3,0.6,0.8,0.9,1\}$. The two-norm of the tracking errors in each iterations is shown in Figure 8. It can be seen that the fastest tracking speed happens as $\alpha=0.8$. 


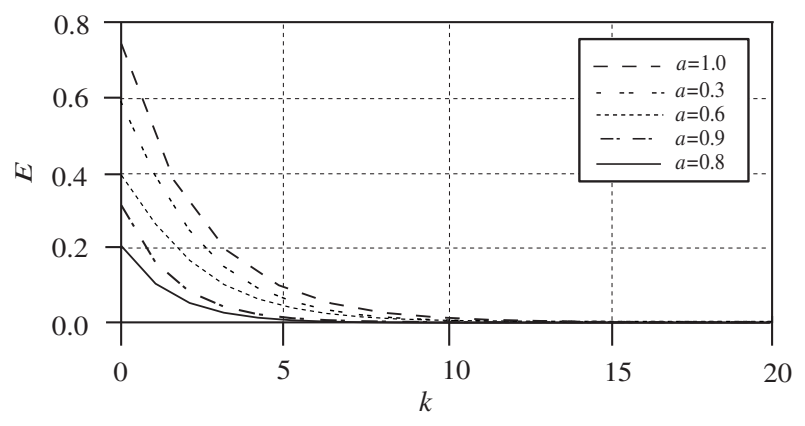

Figure 8. The iterative number-max tracking error with different $\alpha$.

\section{Conclusions}

In this paper, a second order P-type ILC scheme was applied to fractional order distributed parameter systems. The convergent condition was derived using the Gronwall inequality, which has a broader range of applications and is less conservative than some existing ones. The validity of the proposed design method was demonstrated by a numerical example. Our future work includes ILC with initial state learning and D-type ILC. Moreover, the high order ILC law will also be included.

Author Contributions: Y.-H.L. carried out theoretical analysis and wrote the paper. Z.-M.C. performed the experiment and analyzed the data.

Funding: This research was funded by the National Natural Science Foundation of P.R. China grant number 61573298, the Scientific Research Fund of the Hunan Provincial Education Department grant number 15B238 and the Key Laboratory of Intelligent Computing and Information Processing of Ministry of Education.

Conflicts of Interest: The authors declare that there is no conflict of interest regarding the publication of this paper. There have no financial and personal relationships with other people or organizations that would influence our work. There is no professional or other personal interest of any nature or kind in any product, service and/or company that could be construed as influencing the position presented in, or the review of the manuscript.

\section{References}

1. Xu, J.X.; Tan Y. Linear and Nonlinear Iterative Learning Control; Springer: Berlin, Germany, 2003.

2. Ahn, H.S.; Moore, K.L.; Chen, Y.Q. Iterative Learning Control: Robustness and Monotonic Convergence for Interval Systems; Springer: London, UK, 2007.

3. Ardakani, M.M.G.; Khong, S.Z.; Bernhardsson, B. On the convergence of iterative learning control. Automatica 2017, 78, 266-273. [CrossRef]

4. Chi, R.; Huang, B.; Wang, D.; Zhang, R.; Feng, Y. Data-driven optimal terminal iterative learning control with initial value dynamic compensation. IET Control Theory Appl. 2016, 10, 1357-1364. [CrossRef]

5. Li, Y.; Chen, Y.Q.; Ahn, H.S. Fractional order iterative learning control for fractional order linear systems. Asian J. Control 2011, 13, 54-63. [CrossRef]

6. Li, F.; Zhang, K.; Chai, Y.; Xu, S.; Yang, Z. Iterative learning fault estimation design for nonlinear system with random trial length. Complexity 2017, 2017, 1850737.

7. Chani-Cahuana, J.; Landin, P.N.; Fager, C.; Eriksson, T. Iterative learning control for RF power amplifier linearization. IEEE Trans. Microw. Theory Tech. 2017, 64, 2778-2789. [CrossRef]

8. Christofides, P.D. Nonlinear and Robust Control of PDE Systems: Methods and Applications to Transport-Reaction Processes; Birkhauser: Boston, MA, USA, 2001.

9. Jiang, Z.; Cui, B.; Wu, W.; Zhuang, B. Event-driven observer-based control for distributed parameter systems using mobile sensor and actuator. Syst. Control Lett. 2013, 62, 70-76. [CrossRef]

10. Demetriou, M.A. Synchronization and consensus controllers for a class of parabolic distributed parameter systems. Comput. Math. Appl. 2016, 72, 2854-2864. [CrossRef]

11. Li, X.; Mao, W. Finite-time stability and stabilisation of distributed parameter systems. IET Control Theory Appl. 2017, 11, 640-646. [CrossRef] 
12. Choi, J.H.; Seo, B.J.; Lee, K.S. Constrained digital regulation of hyperbolic PDE systems: A learning control approach. J. Chem. Eng. 2001, 18, 606-611. [CrossRef]

13. Chao, X.; Arastoo, R.; Schuster, E. On iterative learning control of parabolic distributed parameter systems. In Proceedings of the 17th Mediterranean Conference on Control Automation, Thessaloniki, Greece, 24-26 June 2009; pp. 510-515.

14. Huang, D.Q.; Xu, J.X. Steady-state iterative learning control for a class of nonlinear PDE processes. J. Process Control 2011, 21, 1155-1163. [CrossRef]

15. Dai, X.S.; Tian, S.P. Iterative learning control for distribute parameter systems with time-delay. In Proceedings of the 2011 Chinese Control and Decision Conference, Mianyang, China, 23-25 May 2011; pp. 2304-2307.

16. Kang, J. A newton-type iterative learning algorithm of output tracking control for uncertain nonlinear distributed parameter systems. In Proceedings of the 33rd Chinese Control Conference, Nanjing, China, 28-30 July 2014; pp. 8901-8905.

17. Huang, D.Q.; Xu, J.X.; Li, X.F.; Xu, C.; Yu, M. D-type anticipator iterative learning control for a class in homogeneous heat equations. Automatica 2013, 49, 2397-2408. [CrossRef]

18. Huang, D.; Li, X.F.; Xu, J.X.; Xu, C.; He, W. Iterative learning control of inhomogeneous distributed parameter systems frequency domain design and analysis. Syst. Control Lett. 2014, 72, 22-29. [CrossRef]

19. Dai, X.S.; Tian, S.P.; Peng, Y.J.; Luo, W.G. Closed-loop P-type iterative learning control of uncertain linear distributed parameter systems. IEEE/CAA J. Autom. Sin. 2014, 1, 267-273.

20. Xiao, T.; Li, H.X. Eigenspectrum-based iterative learning control for a class of distributed parameter system. IEEE Trans. Autom. Control 2017, 62, 824-836. [CrossRef]

21. Chen, Y.Q.; Gong, Z.M.; Wen, C.G. Analysis of a high order iterative learning control algorithm for uncertain nonlinear systems with state delays. Automatica 1998, 34, 345-353. [CrossRef]

22. Sun, M.; Wang, D.; Wang, Y. Varying order iterative learning control against perturbed initial conditions. J. Franklin Inst. 2010, 347, 1526-1549. [CrossRef]

23. Lan, Y.H.; Zhou, Y. $D^{\alpha}$-type iterative learning control for fractional order linear time-delay systems. Asian J. Control 2013, 15, 669-677. [CrossRef]

24. Dai, X.S.; Tian, S.P.; Luo, W.; Guo, Y. Iterative learning control with forgetting factor for linear distributed parameter systems with uncertainty. J. Control Sci. Eng. 2014, 2014, 508573. [CrossRef]

25. Podlubny, I. Fractional Differential Equations; Academie Press: New York, NY, USA, 1999.

26. Hilfe, R. Application of Fractional Calculus in Physics; World Science Publishing: Singapore, 2000.

27. Wharmby, A.W.; Bagley, R.L. The application of the fractional calculus model for dispersion and absorption in dielectrics I. Terahertz waves. Int. J. Eng. Sci. 2015, 93, 1-12. [CrossRef]

28. Ninghu, S.; Paul, N.N.; Sarah, C. The distributed order fractional diffusion-wave equation of groundwater flow: Theory and application to pumping and slug tests. J. Hydrol. 2015, 529, 1262-1273.

29. Atanackovic, T.M.; Opranica, L.; Pilipovic, S. On a nonlinear distributed order fractional differential equation. J. Math. Anal. Appl. 2007, 328, 580-608. [CrossRef]

30. Raheem, A.; Maqbul, M. Oscillation criteria for impulsive partial fractional differential equations. Comput. Math. Appl. 2017, 73, 1781-1788. [CrossRef]

31. Gorenflo, R.; Luchko, Y.; Stojanovic, M. Fundamental solution of a distributed order time fractional diffusion wave equation as a probability density. Fract. Calc. Appl. Anal. 2013, 16, 297-316. [CrossRef]

32. Khader, M.M.; El Danaf, T.S.; Hendy, A.S. A computational matrix method for solving systems of high order fractional differential equations. Appl. Math. Model. 2013, 37, 4035-4050. [CrossRef]

33. Ren, J.; Sun, Z.Z. Maximum norm error analysis of difference schemes for fractional diffusion equations. Appl. Math. Comput. 2015, 256, 299-314. [CrossRef]

34. Morgado, M.L.; Ren, M.R. Numerical approximation of distributed order reaction Cdiffusion equations. J. Comput. Appl. Math. 2015, 275, 216-227. [CrossRef]

35. Duarte-Mermoud, M.A.; Aguila-Camacho, N.; Gallegos, J.A.; Castro-Linare, R. Using general quadratic Lyapunov functions to prove Lyapunov uniform stability for fractional order systems. Commun. Nonlinear Sci. Numer. Simul. 2015, 22, 650-659. [CrossRef]

36. Ye, H.; Gao, J.; Ding, Y. A generalized Gronwall inequality and its application to a fractional differential equation. J. Math. Anal. Appl. 2007, 328, 1075-1081. [CrossRef]

37. Li, J.; Li, J. Adaptive fuzzy iterative learning control with initial-state learning for coordination control of leader-following multi-agent systems. Fuzzy Sets Syst. 2014, 248, 122-137. [CrossRef] 
38. Lan, Y.H. Iterative learning control with initial state learning for fractional order nonlinear systems. Comput. Math. Appl. 2012, 64, 3210-3216. [CrossRef]

39. Bateman, H. Partial Differential Equations of Mathematical Physics; Cambridge University Press: Oxford, UK, 1932.

40. Babaei, D.; Armaou, P.A. Adaptive control of chemical distributed parameter systems. Ifac Papersonline 2015, 48, 681-686. [CrossRef]

41. Shen, L.; He, J.J.; Yu, S.Y. Temperature control for thermal treatment of aluminum alloy in a large-scale vertical quench furnace. J. Cent. South Univ. 2016, 23, 1719-1728. [CrossRef]

42. Huang, L.; Wang, L.; Shi, D. Discrete fractional order chaotic systems synchronization based on the variable structure control with a new discrete reaching-law. IEEE/CAA J. Autom. Sin. 2016, 99, 1-7. [CrossRef]

43. Trigeassou, J.C.; Maamri, N.; Sabatier, J.; Oustaloup, A. State variables and transients of fractional order differential systems. Comput. Math. Appl. 2012, 64,3117-3140. [CrossRef]

44. Sumelka, W.; Voyiadjis, G.Z. A Hyperelastic fractional damage material model with memory. Int. J. Solids Struct. 2017, 124, 151-160. [CrossRef]

45. Lan, Y.H.; Zhou, Y. Non-fragile observer-based robust control for a class of fractional order nonlinear systems. Syst. Control Lett. 2013, 62, 1143-1150. [CrossRef]

(C) 2018 by the authors. Licensee MDPI, Basel, Switzerland. This article is an open access article distributed under the terms and conditions of the Creative Commons Attribution (CC BY) license (http:/ / creativecommons.org/licenses/by/4.0/). 